\title{
Valorando las actividades de enfermería en el control prenatal desde la experiencia de la gestante
}

\author{
Assessing nursing activities in prenatal control from pregnant experience
}

Nayibe Guerra ${ }^{1}$, Rosa Reina ${ }^{2}$, María Hilda Cárdenas ${ }^{2}$, Flor Sanmiguel ${ }^{2}$

\section{RESUMEN}

Objetivo: Interpretar la valoración de las actividades de enfermería en el control prenatal desde la experiencia de la gestante. Materiales y Métodos: La investigación es de enfoque cualitativo, fenomenológico hermenéutico. Como técnica de recolección de información que se emplea es la entrevista a profundidad, mediante encuentros directos con los 2 actores claves con seudónimos de agua y sol. Para el desarrollo de este estudio y el análisis de datos fue el método de Spiegelberg que permite estudiar el campo de la conciencia y explorar a las personas en estudio, partiendo de sus vivencias. Resultados: Del análisis de los resultados se derivan 2 categorías: Acciones de enfermería sentido de compromiso y Preocupación, factores de riesgos más frecuentes. De estas categorías se puede deducir que el cuidado prenatal es de suma importancia porque permite la identificación de riesgos durante el parto y así evitar las complicaciones durante su desarrollo, en donde destacan como causas de mortalidad materna las enfermedades durante su gestación. Conclusiones: Como consideraciones finales se refleja la necesidad de un acercamiento que establezca un ambiente más abierto que permita que se dé encuentros de familiaridad, confianza, respeto por el otro e ir más allá de las actividades exigidas por la institución. Además, la esencia que da enfermería a la importancia del cuidado del binomio madre e hijo en el control prenatal; esto da una apertura hacia la humanización del cuidado en su más amplio significado, ya que existen expresiones por el respeto a la vida y a la condición humana.

Palabras claves: valorando, actividades, enfermería, control prenatal, experiencia, gestante

\begin{abstract}
Objective: To interpret the assessment of nursing activities in prenatal care from the pregnant woman's experience. Materials and Methods: The research is qualitative, hermeneutical phenomenological approach. As an information gathering technique that is used is the in-depth interview, through direct meetings with the 2 key actors with pseudonyms of water and sun. For the development of this study and data analysis was the Spiegelberg method that allows to study the field of consciousness and explore the people in study, based on their experiences. Results: From the analysis of the results, 2 categories are derived: Nursing actions sense of commitment and concern, most frequent risk factors. From these categories it can be deduced that prenatal care is of the utmost importance because it allows the identification of risks during childbirth and thus avoid complications during its development, where diseases during pregnancy stand out as causes of maternal mortality. Conclusions: The final considerations reflect the need for an approach that establishes a more open environment that allows encounters of familiarity, trust, respect for each other and go beyond the activities required by the institution. In addition, the essence that gives nursing to the importance of caring for the mother and child in prenatal control; this gives an opening towards the humanization of care in its broadest sense, since there are expressions of respect for life and the human condition.
\end{abstract}

Keywords: assessing, activities, nursing, prenatal control, experience, pregnant

${ }^{1}$ Coordinación Red Atención Primaria ASIC Campo de Carabobo Municipio Libertador Edo. Carabobo-Venezuela. ${ }^{1}$ Universidad de Carabobo. Facultad de Ciencias de la Salud. Escuela de Enfermería. Venezuela.

\section{INTRODUCCIÓN}

El cuidado exige destrezas y capacidades con conocimientos para establecer vínculos que sean apropiados con las gestantes, orientados a la atención y promoción de la salud. Del mismo modo enfoca su mirada entorno a este concepto, el cual nos hemos convertido en disciplina, sin duda uno de los grandes retos de la enfermería ha sido definir significados. Percibida la importancia 
de la vida fetal, se debería cuidar de la salud de la gestante, no solo física, sino también mental. Donde hay que preservar al binomio madre e hijo, en su buen desarrollo y el embarazo llegue a su feliz término.

El cuidado prenatal es de suma importancia porque permite la identificación de riesgos durante el parto y así evitar las complicaciones durante su desarrollo, en donde destacan como causas de mortalidad materna las enfermedades durante su gestación. Para garantizar una correcta ejecución del cuidado en el control prenatal en la APS es imprescindible disponer de un sistema que permita registrar toda la información relevante para una correcta planificación de atención.

Según la OMS, La enfermería abarca la atención autónoma y en colaboración dispensada a personas de todas las edades, familias, grupos y comunidades, enfermos o no, y en todas circunstancias. Comprende la promoción de la salud, la prevención de enfermedades y la atención dispensada a enfermos, discapacitados y personas en situación terminal. (1) Del mismo modo enfermería permite hacer referencia de distintos conceptos relacionados con la salud y es autónoma de prestación de cuidado.

Para Watson, la práctica cotidiana de la enfermería debe sostenerse sobre un solidó sistema de valores humanísticos que la enfermería tiene que cultivar a lo largo de la vida profesional y además integrar los conocimientos científicos para guiar la actividad. Esta asociación humanística científica constituye a la esencia de la disciplina enfermera a partir de los factores curativos relacionados con la ciencia de cuidar. Está centrada en las relaciones de cuidado transpersonal. Dice que la enfermería es un arte cuando la enfermera/o comprende los sentimientos del otro es capaz de detectar y sentir estos sentimientos y expresarlos como la experiencia de la otra persona. En referencia a la afirmación del autor enfermería integra sus conocimientos al cuidado humanizado, en relevancia a la investigación la enfermera en el control prenatal establece su atención al cuidado de la gestante sostenido en valores de solidaridad, comprensión, respeto, ayuda, orientándola y preparándola para recibir la llegada de ese ser tan amado (2).

Según la OMS, define el embarazo como los nueve meses durante los cuales el feto se desarrolla en el útero de la mujer es para la mayoría de las mujeres un período de gran felicidad. Sin embargo, durante el embarazo, tanto la mujer como su futuro hijo se enfrentan a diversos riesgos sanitarios (3). Para enfermería el cuidado de la gestante está enmarcado en la experiencia que tenga en la atención de la consulta prenatal, ya que los conocimientos son clave para garantizar una atención de calidad.

Por tal razón, la consulta prenatal es definida como, un conjunto de acciones médicas y asistenciales que se concretan en entrevistas o visitas programadas con el equipo de salud, a fin de controlar la evolución del embarazo y obtener una adecuada preparación para el parto y la crianza del recién nacido con la finalidad de disminuir los riesgos de este proceso fisiológico. (4) En este orden enfermería realiza una serie de actividades programadas para la atención, promoción y prevención del cuidado de las embarazadas de forma personalizada para su atención, a fin de llevar registro del desarrollo y evolución de su gestación.

En este orden, Suárez, en el 2014 en Colombia, trabajo con la percepción que tienen las gestantes del cuidado brindado por los profesionales de Enfermería a través del curso de preparación para la maternidad y la paternidad realizado en la E.S.E. San Cristóbal en la ciudad de Bogotá. Cuyo propósito fue describir la percepción que tienen las gestantes del cuidado brindado por los profesionales de Enfermería a través del curso de preparación para la maternidad y la paternidad. La metodología usada fue el diseño cualitativo, descriptivo, exploratorio, realizado mediante la técnica de análisis de contenido de Laurence Bardin por medio de una hermenéutica controlada a las entrevistas semiestructuradas de 14 gestantes que asisten al curso de preparación para la maternidad y la paternidad. Teniendo como resultado el tema central "El cuidado de enfermería inmerso, presente y latente en el curso de preparación" que surge del análisis de las categorías, se encuentra que las gestantes se sienten cuidadas por las enfermeras en el curso de preparación teniendo en cuenta tres aspectos fundamentales:

Acompañamiento, reconocimiento y enseñanza en cuidado (5).

El cuidado de enfermería hace parte fundamental del curso de preparación, que al ser dictado sin intervenciones de enfermería se convierte en un taller más y en una clase magistral que no representa ningún valor en las participantes y no trasciende en la experiencia de la gestación (5). El presente estudio da relevancia a la investigación denotando que las gestantes necesitan de enseñanza para su propio cuidado y enfermería debe abocarse a su asistencia y control continuo para que las gestantes al final de su gestación el embarazo llegue a su feliz término y continuación del cuidado mediante su planificación familiar. El objetivo de esta investigación fue interpretar la valoración de las 
actividades de enfermería en el control prenatal desde la experiencia de la gestante.

Desde la mirada de Swanson, el cuidado profesional está representado en su escala de cuidado en dos sub escalas; a) Sanador compasivo: Se refiere al comportamiento del profesional de enfermería que en la práctica del cuidado de la salud de los seres humanos establece una relación afectuosa y de confianza; b) Sanador Competente: Se refiere al comportamiento del profesional de enfermería, con responsabilidad, compromiso, pericia e idoneidad para ejercer la práctica del cuidado de la salud de los seres humanos (6).

Procesos de Cuidado: Identificando los soportes filosóficos empleados en el modelo, es de alta importancia mencionar los procesos en los que describen el modelo de los cuidados; a) Conocer: El Conocimiento o búsqueda es un esfuerzo por entender el significado de un suceso tal cual en la vida del otro, desde lo emocional, desde sus capacidades y desde sus conocimientos; centrándose en la persona que se cuida, buscando claves, valorando meticulosamente y buscando un proceso de compromiso entre el que cuida y el que es cuidado, para que se fortalezca éste proceso; b) Estar con: La compañía es estar emocionalmente presente en el otro, que la persona perciba que estamos disponibles para aclarar sus dudas y enseñarle lo que ella necesita hasta que se empodere del cuidado. Ello implica compromiso, estar ahí, capacidad de comunicación, compartir sentimientos sin abrumar y transmitir disponibilidad; c) Hacer por o para: Hacer por otros lo que haríamos por nosotros mismos si fuera posible, incluyendo adelantarse a las necesidades, confortar, actuar con habilidad y competencia y proteger al que es cuidado, respetando su dignidad.

También implica que en nuestro actuar hagamos las cosas bien, informemos $y$ expliquemos de la mejor manera y a su vez reconfortemos al otro; d) Permitir o posibilitar: Es ayudar a, o facilitar el paso del otro por transiciones de la vida o por situaciones desconocidas. Este proceso incluye las educativas que utilizamos para que las personas aprendan a cuidarse. Aquí es necesario generar alternativas, retroalimentar permanentemente, hacer seguimiento y evaluar o validar lo que se ha aprendido; e) Mantener las creencias: Mantener la fe o la creencia de que el otro tiene la capacidad de salir adelante, que va a superar un hecho o acontecimiento y se va a enfrentar al futuro con un significado, teniéndole en alta estima, manteniendo una actitud llena de esperanza, ofreciendo un optimismo realista y ayudando a encontrar ese significado estando al lado de la persona cuidada en cualquier situación (6).

En este orden Watson afirma que la enfermera debe comprender a la persona como ser dotado de creencias, emociones, sentimientos y temores, sin olvidar su propia individualidad y su conocimiento; debe comprender y sentir profundamente las necesidades de su paciente para poder satisfacerlas, por ser una teoría interaccionista puede ser referente para el perfeccionamiento de las relaciones enfermera gestante, fortaleciendo la identidad profesional y creando nuevas perspectivas para una enfermería ética y humana sin perder la visión de la ciencia. La aproximación de esta teórica al cuidado de enfermería se basa en tres premisas fundamentales (2).

\section{MATERIALES Y MÉTODOS}

La investigación está dentro del paradigma cualitativo, con un enfoque fenomenológico hermenéutico. Como técnica de recolección de información que se emplea es la entrevista a profundidad, mediante encuentros directos con los 2 actores claves con seudónimos de agua y sol. Para el desarrollo de este estudio y el análisis de datos fue el método de Spiegelberg que permite estudiar el campo de la conciencia y explorar a las personas en estudio, partiendo de sus vivencias.

\section{RESULTADOS Y DISCUSIÓN}

Del análisis de las entrevistas surgieron 2 categorías:

Acciones de enfermería sentido de compromiso Agua: Hacen historia médica, revisan ecografía y exámenes de laboratorio, controlan peso, talla tensión arterial, dan charlas educativas alimentación, vestimenta, vitaminas y cuidado en el hogar, dan consejos.

Sol: Hacen historia, Solicitan copia de ecografías, exámenes de laboratorios, pesan, tallan toman tensión, vacunación y charlas de cuidado, alimentación, vestido y caminar.

\section{Preocupación, factores de riesgos más frecuentes}

Agua: La tensión alta, las infecciones urinarias, anemia, el aborto, el estrés, preocupación por no tener dinero.

Sol: Anemias, infecciones urinarias, amenazas de aborto, tensión alta, sangrado, desnutrición. 
Según los Protocolos de Atención en Cuidados Prenatales y Atención Obstétrica de Emergencia (2014) Se realiza anamnesis y evaluación general, estimación de la edad de gestación y fecha probable de parto. Se solicitan exámenes de rutina. Se evidencia las acciones protocolizadas de la enfermera en las actividades de la consulta prenatal y que son vistas por las usuarias como actividades que denotan la responsabilidad de la enfermera.

\section{CONCLUSIONES}

Se refleja la necesidad de un acercamiento que establezca un ambiente más abierto que permita que se dé encuentros de familiaridad, confianza, respeto por el otro e ir más allá de las actividades exigidas por la institución. La esencia que da enfermería a la importancia del cuidado del binomio madre e hijo en el control prenatal; esto da una apertura hacia la humanización del cuidado en su más amplio significado, ya que existen expresiones por el respeto a la vida y a la condición humana.

\section{REFERENCIAS BIBLIOGRÁFICAS}

1. Organización Mundial de la Salud. Temas de Salud: Enfermería. 2015. Consultado: Mayo 2016 https://www.who.int/topics/nursing/es/

2. Tomey, Ann Marriner y Alligood, Marhta Raile. Modelos y Teorias de Enfermeria. 2010 sexta edicion.

3. OMS.http://www.who.int/topics/pregnancy/ es/. 2015.

4. MSDS. Protocolos de Atencion en Cuidados Prenatales y Atencion Obstetrica de Emergencia. 17 de Abril de 2014.

5. Suáres D. Percepción del Cuidado de Enfermería de las Gestantes uqe Asisten al Curso Maternidad y Paternidad un Proyecto de Vida. Colombia - Bogóta : Universidad Nacional de Colombia Facultad de Enfermeria, 2014.

6. Gualdron, Lucy Maricela Vesga. Validez y Confiabilidad de la Escala de Cuidado Profesional (CPS) de la Dra. Kristen SWason. 2013. 